\title{
Image recognition algorithm undecimated wavelet transform coupling moment
}

\author{
Liu Hongli ${ }^{1, a}$, Ye Liying ${ }^{1, a}$ \\ ${ }^{1}$ Computer Engineering College, Jimei University, 361021, China \\ ${ }^{2}$ School of Science, Jimei University, 361021, China \\ aemail:liuhongli_fj@163.com
}

\begin{abstract}
Keywords: Undecimated Wavelet Transform; Zernike Moment; Copy And Paste; Image Forgery Detection; Robustness
\end{abstract}

\begin{abstract}
In order to solve the problem that robustness in copying and pasting forgery detection algorithm is poor and detection accuracy is insufficient, this paper puts forward to image forgery detection algorithm of coupling Zernike Moment (Zernike) by Undecimated Wavelet Transform (UWT). Firstly, it uses Undecimated Wavelet Transform to break the input image up into approximation coefficient LL and specific coefficient $\mathrm{HH}$ and get similarity and difference among blocks; then it divides overlapping block and calculate space between image subblock, makes order and classification according to similarity LL and difference $\mathrm{HH}$ and introduces Zernike Moment, builds distance matrix for image feature matching and image forgery detection. The experiment indicates that the mentioned algorithm has good translation invariance and amplitude rotation invariance and it has good anti-post processing performance, high accurate measurement, low omission factor and false positive rate.
\end{abstract}

\section{Introduction}

In the aspect of image forgery, copying-moving falsification is one of the common methods ${ }^{[3]}$, but this algorithm is matching on the basis of similar image blocks. If every block of detected image has certain similarity, it will be misidentified as copy-move area so as to cause failure of true copy-move area to eliminate and produce the possible false detection. CHRISTLEI, ${ }^{[5]}$ proposes falsification detection of algorithm based on image pixel matching. The experiment shows that the complication of such algorithm is reduced, easily eliminates JPEG compression impact and has highly accurate location for copy-paste falsification area. However, if copy and paste area is located among two or above image blocks, the detection performance of such algorithm is greatly reduced. Mahdian ${ }^{[6]}$ makes reasonable hypothesis for copy and falsification of area. They design a brand-new detection technology, but the time consumption of this algorithm at the time of detection is serious, and it cannot complete detection of multiple falsification areas in target image.

For this, the paper designs copy-move image forgery detection algorithm of coupling Zernike Moment (Zernike) by Undecimated Wavelet Transform (UWT). Through introducing Zernike Moment, this paper constructs distance matrix for image feature matching and image forgery detection. At last, this paper verifies the detection property of forgery location technique.

\section{Forgery Detection Algorithm of this Paper}

Wavelet Transform (WT) has the feature of good time-frequency localization and it's popular in image processing and signal analysis field. In pattern recognition, translation invariance of signal is vital. When signal is translated, the relative value should be translated rather than changed. Image characteristic quantity can be got from discrete wavelet transform (DWT) through transformation. Utilizing flexible translation to break down signal in multiple scales can adapt to the requirement of signal analysis better to get signal information in details. The downsampling adopted by DWT destroys the translation invariance of wavelet transform to make DWT sensible to initial position of signal, thus the tiny amount of movement for time domain and space domain may cause great change of wavelet transform coefficient ${ }^{[8]}$. 
The technology based on Undecimated Wavelet Transform proposed by this paper firstly breaks down UWT of image, divides overlapping block, extracts low-frequency component (fuzzy information) LL and high-frequency component (specific information) $\mathrm{HH}$, and then calculates similarity and difference of every blocking respectively and gets distance of every blocking; according to the calculated distance, makes ascending order of LL result and descending order of $\mathrm{HH}$ result; uses the combination of similarity of LL and difference of $\mathrm{HH}$ for judgment and introduces Zernike Moment to construct distance matrix for image feature matching and image forgery detection. See Fig. 1 for operation process of algorithm in this paper.

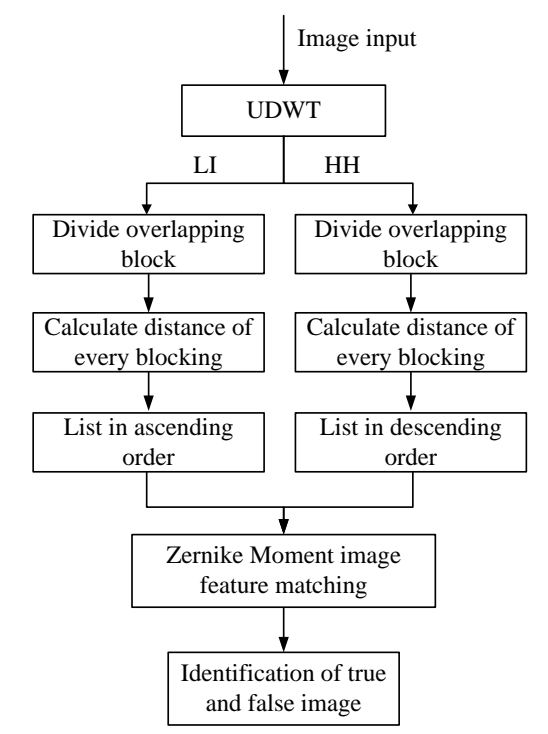

\section{Undecimated wavelet transform}

Fig.1. Algorithm process chart of this paper

For image identification, signal feature must keep translation invariance, because when a image area moves, the feature will move correspondingly rather than be damaged [9]. For example, for falsification of copy-move image, it will not be damaged in such two similar areas as copy area and paste area. If non-translation is invariable, two areas will appear different manifestations, so missing detection may appear for forgery detection. In DWT, the existence of downsampling doesn't have translation invariance, as is shown in Formula (1) and (2), so calculate wavelet coefficient and get factor of every direction through convolution of downsampling. DWT algorithm doesn't have translation invariance, so small shift of input image may exert huge effect on DWT coefficient in different scales and small spacial shift may produce different feature vector for copy and paste area. Pseudo-Gibbs phenomenon exists in DWT, and the detection effect on signal analysis like edge detection, denosing, texture, etc. is not ideal. In order to overcome the deficiency existed in DWT, this paper proposes UWT algorithm with translation invariance. Because this algorithm excludes downsampling and wavelet coefficient reduction, the transformation is called undecimated.

Set I as input image, $\mathrm{h}(\mathrm{k})$ and $\mathrm{g}(\mathrm{k})$ as low-pass filter and high-pass filter respectively, UWT algorithm of image can be expressed as the following:

Initial conditions: scale $j=0, \mathrm{I}^{0}=\mathrm{I}$, according to Formula (2) and (3), calculate scaling proportion and wavelet coefficient respectively when $j=1,2, \ldots, J$.

$$
\begin{aligned}
& c^{j+1}(n)=\sum_{k} h(k) c^{j}\left(n+2^{j} k\right) \\
& d^{j+1}(n)=\sum_{k} g(k) c^{j}\left(n+2^{j} k\right)
\end{aligned}
$$

$h^{j}(k)$ and $h^{j}(k)$ are result of $h(k)$ and $g(k)$ in scale $j$ after filtering, UWT process is as follows:

Original state, $I^{0}=I$, calculate scaling proportion $I^{j}$ and wavelet coefficient $D^{j}$ respectively when $j=1,2, \ldots, J$.

$I^{j-1}$ goes through $h^{j-1}(k)$ filter 
$I^{j-1}$ goes through $g^{j-1}(k)$ filter

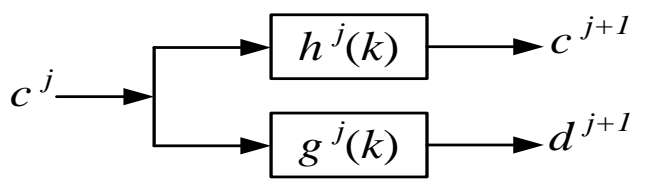

Fig.2. UWT algorithm 1D breakdown diagram

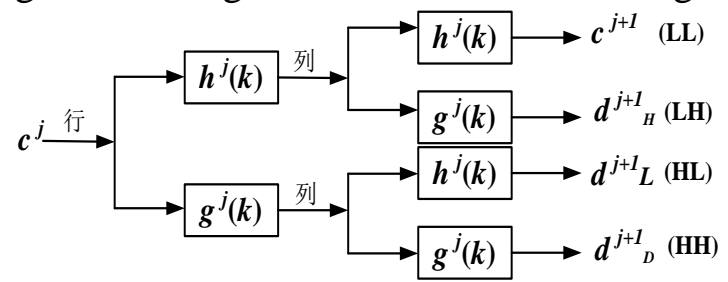

Fig.3. UWT algorithm 2D breakdown diagram

Fig.2 is $1 \mathrm{D}$ breakdown of UWT algorithm. In wavelet transform, $I^{j}$ is called low-pass sub band $(\mathrm{L}), D^{j}$ is high-pass sub band $(\mathrm{H})$. As a kind of $2 \mathrm{D}$ signal, image is made up of four kinds of sub bands that is LL, LH, HL and HH. The size of every sub band is the same as the artwork master. As is shown in Fig.3, utilize UWT to break down 2D image along row and line and get low-pass sub band LL, level high-pass sub band LH, vertical high-pass sub band HL and diagonal high-pass sub band $\mathrm{HH}$.

\section{Zernike Moment}

After extracting image feature through Undecimated Wavelet Transform, it requires to find out the corresponding relation among image blocks for image matching calculation. Image matching is the key procedure in image identification. The effect of common algorithm based on gradation of image in image rotation is not ideal, while image moment invariance is able to eliminate the adverse effect caused by image rotation, so adopt Zernike Moment with image moment invariance as image matching algorithm [10]. Based on orthogonal polynomials, form positive intersection with complete unit circle. The model is defined as follows:

$$
V_{n m}(x, y)=V_{n m}(r, \theta)=R_{n m}(r) e^{j m \theta}
$$

Of them, $(x, y)$ means position of pixel; $n$ is non-negative integer, showing order; $m$ is non-zero integer, showing multiplicity; $r$ means radius, and $\theta$ means angle. Of them: $r=\sqrt{x^{2}+y^{2}}, \theta=\operatorname{acr} \tan \frac{x}{y}, R_{n m}(r)[$ is Zernike radial polynomial, and the definition is as follows:

$$
R_{n m}(r)=\sum_{k=0}^{(n-|m|) / 2} \frac{(-1)^{k}(n-s) !}{k !\left[\frac{n+|m|}{2}-k\right] !\left[\frac{n-|m|}{2}-k\right]} r^{n-2 k}
$$

Thus, 2D Zernike Moment of image function $f(x, y)$ at $n$ order $m$ degree can be defined as follows:

$$
Z_{n m}=\frac{n+1}{\pi} \iint_{x^{2}+y^{2} \leq 1} f(x, y) V^{*}{ }_{n m}(r, y) d x d y
$$

Of them, * means complex conjugate. For digital image, model (7) at polar coordinates can be expressed as follows:

$$
Z_{n m}=\frac{n+1}{\pi} \sum_{r<10<\theta<2 \pi} \sum_{n}(r, \theta) V^{*}{ }_{n m}(r, \theta) r
$$

If rotate the image by $\delta$ degree, then Zernike Moment becomes:

$$
Z_{n m}=\frac{n+1}{\pi} \sum_{r<10<\theta<2 \pi} \sum_{0}(r, \theta) V^{*}{ }_{n m}(r, \theta-\delta) r
$$

After transformation, we get:

$$
\begin{aligned}
& Z^{\prime}{ }_{n m}=Z_{n m} e^{j m \delta} \\
& \left|Z^{\prime}{ }_{n m}\right|=\left|Z_{n m}\right|
\end{aligned}
$$


From model (9) and (10), we can get, Zernike Moment of image has the property of amplitude rotation invariance and strong noise immunity. The specific procedure of image Zernike Moment is as follows:

For every image block of detected image, select a circle with radium as $r$, calculate Zernike Moment of every feature neighborhood.

Construct distance matrix $D$, elements in matrix $D$ are:

$D_{i j}=\sum_{m=1}^{n}\left|p_{i}-p_{j}\right|$

Of them, $P_{i}$ and $P_{j}$ are element of detected image respectively.

In distance matrix $D$, find minimum value in row and line respectively. When $D_{i j}$ reaches minimum value in the row and line at the same time, $P_{i}$ is matched with $P_{j}$ in feature.

\section{Experimental Results}

To verify the effectiveness and superiority of algorithm of this paper, set up two control groups: literature [4] and literature [5] are marked as A algorithm and B algorithm. By virtue of MATLAB8.0 test text algorithm property, simulation condition is: $D E L L ~ I 5,2.3 \mathrm{GHz}$ quad-core CPU, 500GB hard disk, 4GB memory, Windos7 system. In CASIA TIDEV1.0 database [11], select image size $384 \times 256$.

In order to measure algorithm property, introduce omission factor FN, false detection rate FP and accuracy AC to represent [3].

\section{Non-rotary copy and paste forgery detection in falsification area}

Image forgery processing is by virtue of Photoshop CS3software, as is shown in Fig.4. Fig.4 (a) is original image, Fig.4 (a) is copy-paste forgery image, Fig.4 (c) is detection result of standard algorithm, black area means being detected to be copy and paste area, Fig.4 (d) is detection result of A algorithm, of them, the marked red area is false detection area; Fig.4 (e) is detection result of B algorithm, indicating that such algorithm has high omission factor.

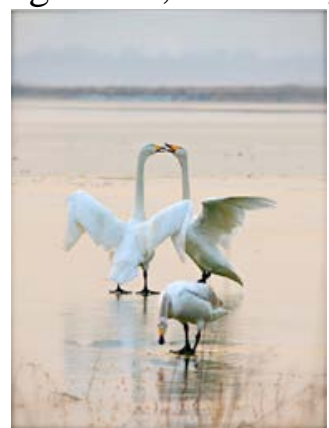

(a) Original image

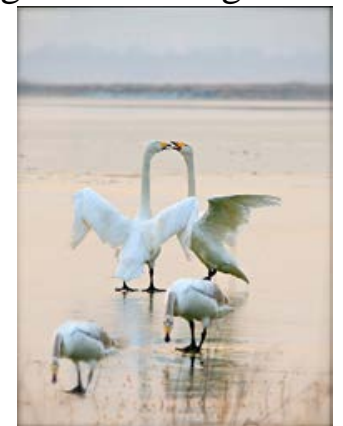

(b) forgery image

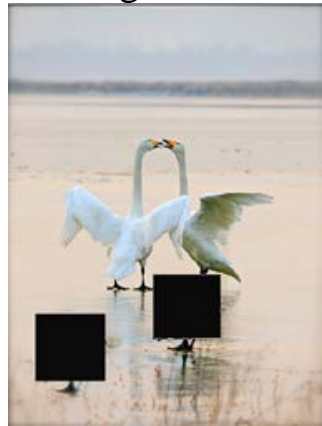

(c) Detection

result of algorithm of this paper

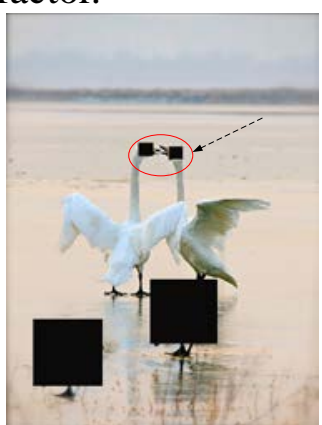

(d) Detection result of A algorithm

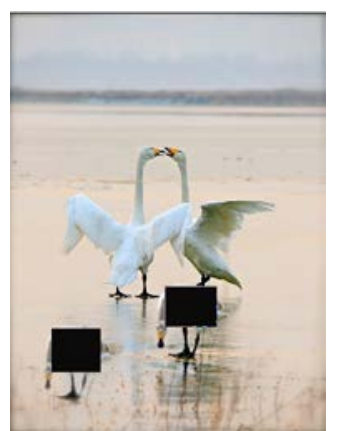

(e) Detection result of B algorithm

Fig.4. Image forgery detection results of A algorithm, B algorithm and algorithm of this paper

Copy image is divided into 384 of $16 \times 16$ image blocks, the same as paste image in quantity, so there are 768 image blocks. By Using three kinds of algorithms to detect image, we get that algorithm of this paper is superior to the other kinds in accuracy, false detection rate and omission factor. Algorithm of this paper has translation invariance UWT, so it will not be damaged when image in copy and paste area and it will protect detailed information in falsification area better. Fig.6 is detection error weighing diagram of three kinds of algorithms. From Fig.6, we can see false detection rate and omission factor of algorithm is very low compared with A algorithm and B algorithm. 


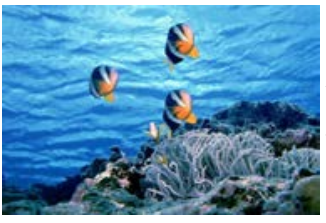

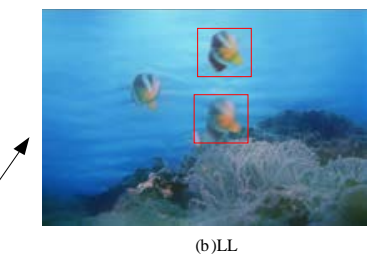

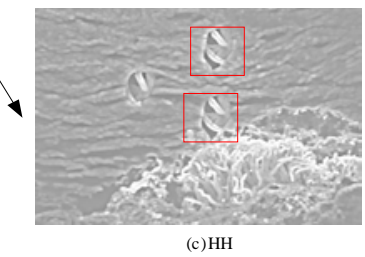

Fig.5. UWT breakdown result of moving forgery image

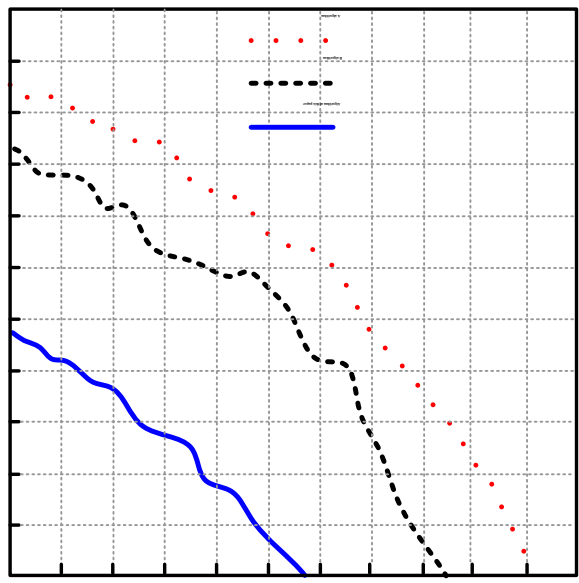

Fig.6. Weighing result of detection error of three algorithms

\section{Conclusion}

This paper proposes a copy and paste image forgery detection technology of coupling Zernike Moment based on Undecimated Wavelet Transform. By using Undecimated Wavelet Transform, this paper gets sub band LL and $\mathrm{HH}$, calculate similarity and difference of image block. By utilizing the combination of similarity of LL and difference of $\mathrm{HH}$, this paper makes dual judgment and introduces Zernike Moment to construct distance matrix for image feature marching. In CASIA TIDEV1.0data, assess the algorithm. Experimental results indicate that: algorithm of this paper has good noise immunity and resistance to compression. It has high accuracy, low omission factor and low false detection rate.

\section{References}

[1] He, J., Geng, Y., Wan, Y., Li, S., and Pahlavan, K. (2013). A cyber physical test-bed for virtualization of RF access environment for body sensor network. Sensors Journal, IEEE, 13(10), 3826-3836.

[2] Lv Z, Tek A, Da Silva F, et al. Game on, science-how video game technology may help biologists tackle visualization challenges[J]. PloS one, 2013, 8(3): 57990.

[3] Su T, Wang W, Lv Z, et al. Rapid Delaunay triangulation for randomly distributed point cloud data using adaptive Hilbert curve[J]. Computers \& Graphics, 2016, 54: 65-74.

[4] Jinyu Hu, Zhiwei Gao and Weisen Pan. Multiangle Social Network Recommendation Algorithms and Similarity Network Evaluation[J]. Journal of Applied Mathematics, 2013 (2013). 